03.1;03.2

\title{
Влияние периодической макрошероховатости на развитие турбулентной свободной конвекции у внезапно нагреваемой вертикальной пластины
}

\author{
(C) А.М. Левченя, Е.М. Смирнов, С.Н. Трунова
}

Санкт-Петербургский политехнический университет Петра Великого, Санкт-Петербург, Россия

E-mail: levchenya_am@spbstu.ru

Поступило в Редакцию 7 сентября 2021 г.

В окончательной редакции 26 октября 2021 г.

Принято к публикации 27 октября 2021 г.

Представлены результаты численного моделирования нестационарной свободной конвекции, развивающейся у внезапно нагреваемой пластины, на которой в шахматном порядке расположены выступы в виде адиабатических цилиндров удвоенной (по отношению к диаметру) высоты. Расчеты выполнены по уравнениям Рейнольдса с применением дифференциальной модели турбулентных напряжений. Определен интервал изменения числа Грасгофа (построенного по толщине свободноконвективного течения), в котором может достигаться значительная (до $80 \%$ ) интенсификация теплоотдачи. Показано, что наилучшие условия для интенсификации создаются, если продольный шаг в массиве выступов примерно в 20 раз превосходит диаметр последних.

Ключевые слова: нестационарная свободная конвекция, пограничный слой на вертикальной пластине, интенсификация теплоотдачи, численное моделирование, модель рейнольдсовых напряжений.

DOI: 10.21883/PJTF.2022.03.51984.19020

При разработке и эксплуатации многих сооружений и технических устройств остро стоит проблема охлаждения нагревающихся поверхностей и соответственно актуален вопрос интенсификации теплообмена. Традиционный подход к интенсификации пристенных процессов теплопереноса заключается в использовании выступов и ребер различной формы [1]. Альтернативой по отношению к использованию выступов является применение активно исследуемых в последние годы углублений в поверхности в форме круглых, овальных и овальнотраншейных лунок (см., например, [2,3]). Влияние выступов и углублений обычно исследуется в условиях вынужденной конвекции. Однако во многих практических приложениях пассивное охлаждение конструкций происходит в условиях свободной конвекции [4], при этом на поверхностях промышленных установок и жилых помещений реальных размеров свободноконвективный пограничный слой находится в турбулентном состоянии.

Особенности теплообмена в свободноконвективном пограничном слое во многом связаны с немонотонностью профиля продольной скорости, в котором выделяются две части: 1) внутренняя - от стенки до точки максимума скорости; 2) внешняя - от максимума до внешней границы с окружающей средой, существенно более протяженная [5]. Как установлено в результате предыдущих исследований, существенная интенсификация теплоотдачи может быть достигнута лишь в том случае, когда возмущается не только внутренняя, но и внешняя часть пограничного слоя [6]. Это можно реализовать, в частности, посредством расположения на нагретой поверхности крупномасштабных препятствий.
Влияние одиночного препятствия в виде кругового цилиндра различной высоты на локальную интенсификацию теплообмена в квазистационарном турбулентном пограничном слое, развивающемся на изотермической вертикальной пластине, исследовалось в работе [7] на основе осредненных по Рейнольдсу уравнений Навье-Стокса, замкнутых по $k-\omega$ SST-модели турбулентности. Для практических приложений более интересен, однако, случай с множеством крупномасштабных препятствий, распределенных по большой части охлаждаемой поверхности, например, периодически. Обтекание свободноконвективным пограничным слоем одиночного поперечного ряда периодически расположенных цилиндров исследовалось численно в работе [8] на основе постановки и вычислительной методики, примененной ранее в [7]. Интенсифицирующее действие массива $V$-образных пластинок на теплоотдачу в свободноконвективном пограничном слое на вертикальной поверхности экспериментально изучалось в работах [9-11], но лишь для случая ламинарного режима течения в возмущаемом слое.

В настоящей работе численным методом исследуется интенсифицирующий эффект цилиндрических выступов, расположенных в шахматном порядке на поверхности вертикальной неограниченной пластины, которая внезапно нагревается относительно внешней среды. Постановка этой модельной трехмерной задачи, в которой развитие динамических и теплообменных процессов по продольной (вертикальной) координате „подменяется“ развитием их во времени, позволяет кардинальным образом уменьшить ресурсоемкость иссле- 


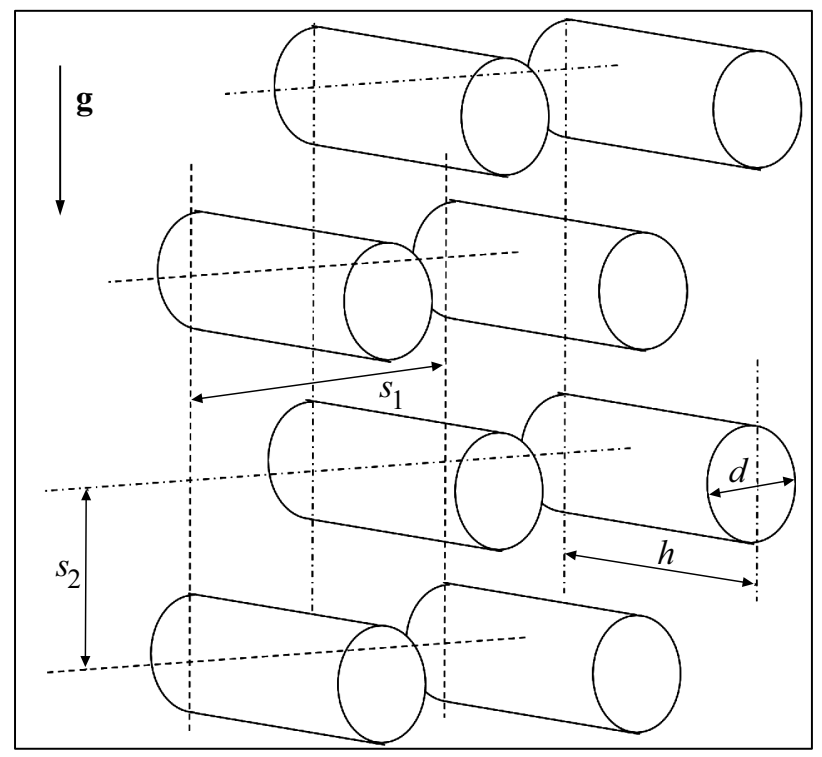

Рис. 1. Схема, поясняющая постановку задачи.

дований вследствие возможности использования компактной расчетной области с наложением условий периодичности по продольному направлению. Отметим, что весьма успешный опыт применения такого подхода для прямого численного моделирования турбулентности в свободноконвективном пограничном слое на гладкой пластине представлен в [12,13]. Решение статистически одномерной нестационарной задачи о развитии свободноконвективного турбулентного пограничного слоя на внезапно нагреваемой гладкой пластине, полученное на основе уравнений Рейнольдса, замкнутых по модели рейнольдсовых напряжений, представлено в работе [14], где показано, в частности, что на стадии развитой турбулентности результаты расчетов хорошо согласуются с данными прямого численного моделирования.

Таким образом, в настоящей работе рассматривается свободноконвективное течение воздуха (число Прандтля $\operatorname{Pr}=0.7)$ в окрестности вертикальной пластины, температура которой $T_{w}$ в начальный момент времени внезапно повышается относительно температуры $T_{a}$ окружающего пространства, заполненного неподвижной средой. На пластине в шахматном порядке расположены цилиндрические выступы диаметром $d$, их поверхность полагается адиабатической. Выступы высотой $h=2 d$ составляют относительно плотные ряды в поперечном направлении с шагом $s_{1}=2 d$ (рис. 1). Продольный шаг $s_{2}$ (расстояние между рядами выступов по вертикальному направлению) варьируется от $6 d$ до $24 d$.

Выбранная для расчетов прямоугольная расчетная область имеет размеры $0.5 s_{1} \times 2 s_{2} \times H$, где $H=50 d-$ протяженность области по нормали к пластине, достаточная для того, чтобы внешняя граница прогреваемого слоя жидкости не достигала границы расчетной области на всем рассматриваемом временно́м участке развития конвекции. На верхней и нижней границах расчетной области ставятся условия периодичности. На боковых границах, проходящих через центры выступов, накладываются условия симметрии. На поверхностях пластины и выступов ставятся условия прилипания.

Используя для скорости в качестве масштаба скорость плавучести, вводимую как $V_{b}=\left[g \beta\left(T_{w}-T_{\alpha}\right) v\right]^{1 / 3} \quad[5]$, определим масштаб времени для рассматриваемой задачи как $t_{s}=d / V_{b}$. Процесс развития конвекции рассчитывается в течение промежутка времени, составляющего $32 t_{s}$. Отметим, что для случая гладкой пластины характерное число Грасгофа $\mathrm{Gr}_{\delta}=g \beta\left(T_{w}-T_{a}\right) \delta^{3} / v^{2}[8]$ в конце этого промежутка составляет $10^{9}$. Здесь $g-$ ускорение свободного падения, $\beta$ - коэффициент теплового расширения, $v-$ кинематический коэффициент вязкости, $\delta$ - интегральная толщина пограничного слоя, вычисляемая посредством интегрирования профиля вертикальной скорости [12] (по величине она близка к полуширине внешней части слоя).

Для получения численного решения применяются нестационарные трехмерные уравнения Рейнольдса и энергии, эффекты плавучести моделируются в приближении Буссинеска. Уравнения замыкаются по модели рейнольдсовых напряжений в BSL-версии, согласно которой расчет временно́го масштаба турбулентности, необходимого для замыкания системы уравнений переноса рейнольдсовых напряжений, проводится по (одно-

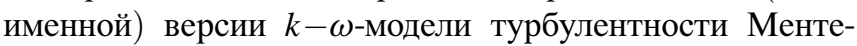
pa [15]. Расчеты по методу конечных объемов выполнялись с использованием гидродинамического кода общего назначения ANSYS Fluent 18.2 на псевдоструктурированных сетках размерностью около двух миллионов ячеек. Выбор числа ячеек и топологии использованных расчетных сеток был основан на результатах исследования сеточной сходимости при численном решении задачи о возмущении свободноконвективного пограничного слоя одиночным цилиндром [7]. Сетки сгущались к твердым стенкам так, чтобы в течение всего рассматриваемого промежутка времени средняя величина $y^{+}$для ближайших к стенкам расчетных точек не превышала единицы. Дискретизация по времени и пространству для всех уравнений переноса осуществлялась по схемам второго порядка. Для продвижения по времени применялся безытерационный солвер, шаг по безразмерному времени составлял 0.004 .

На рис. 2 с использованием полутоновой заливки показаны рассчитанные при $s_{2}=6 d$ и $18 d$ изолинии мгновенного поля безразмерной вертикальной скорости в плоскости симметрии и распределения коэффициента теплоотдачи на пластине в один из моментов времени $\left(t=14.6 t_{s}\right)$, соответствующих развитой турбулентности в моделируемом свободноконвективном течении (коэффициент теплоотдачи $\alpha$ нормирован на значение, вычисленное для того же момента времени в случае гладкой пластины; при визуализации рассчитанное распределение $\alpha$ дублировано с зеркальным отражением). Видно, что в области сочленения выступов и пластины 

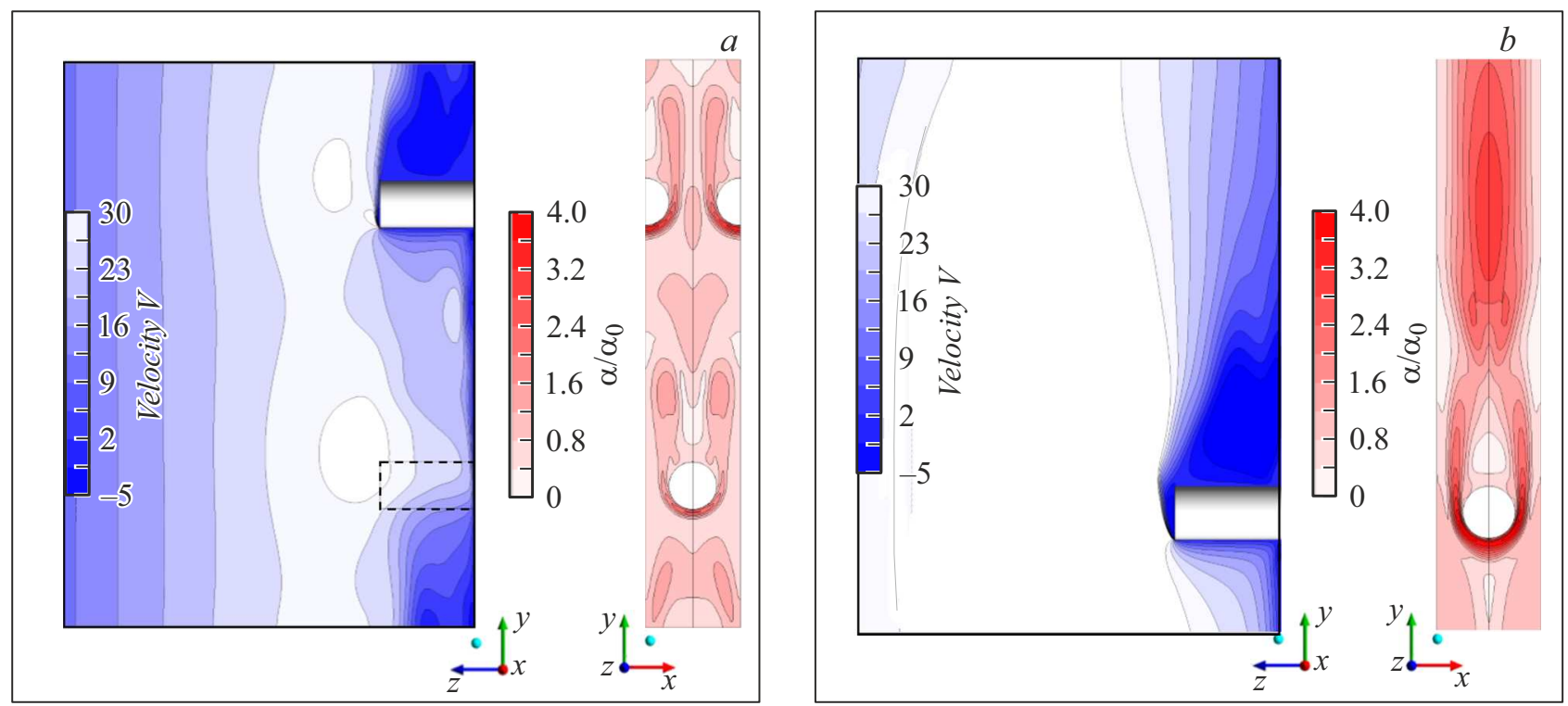

Рис. 2. Поле вертикальной скорости и нормированный коэффициент теплоотдачи на пластине в момент времени $t=14.6 t_{s}$. $s_{2}=6 d(a)$ и $18 d(b)$.

формируются отрывные зоны и крупномасштабные вихревые структуры - как подковообразные, расположенные у основания выступов, так и „следовые“. Наличие подковообразных вихрей обусловливает существенную неоднородность локальной теплоотдачи в окрестности цилиндров. В следе за выступами (на расстояниях более $2 d$ от задней кромки) возникают области повышенной теплоотдачи, протяженность которых и их вклад в интенсификацию теплоотдачи увеличиваются с ростом продольного шага решетки. При $s_{2} / d$, меньших примерно 10, в частности при $s_{2}=6 d$, взаимодействие потока с выступами следующего ряда препятствует формированию протяженной следовой области. Это в сочетании с общим тормозящим действием выступов, увеличивающимся при уменьшении шага, приводит к потере эффекта интенсификации.

Существенные изменения в картине течения, происходящие с ростом толщины прогреваемого слоя, предопределяют сложное поведение во времени интегральной теплоотдачи. Для приближенного переноса полученных результатов на практически интересный случай квазистационарного пограничного слоя, развивающегося вдоль вертикальной пластины с системой выступов, целесообразно рассмотреть изменения интегральной теплоотдачи в зависимости от характерного числа Грасгофа $\mathrm{Gr}_{\delta}(t)$. На рис. 3 рассчитанные при различных значениях $s_{2}$ зависимости осредненного по поверхности пластины коэффициента теплоотдачи $\alpha_{m}\left(\mathrm{Gr}_{\delta}\right)$ сопоставляются с данными, полученными для гладкой пластины по той же верифицированной методике, что и в упомянутой выше работе [14]. Здесь следует отметить, что к данным, полученным при $\mathrm{Gr}_{\delta}<10^{6}$, нужно относиться с осторожностью, так как примененная модель турбулентности не призвана адекватно описывать явления ламинарно-

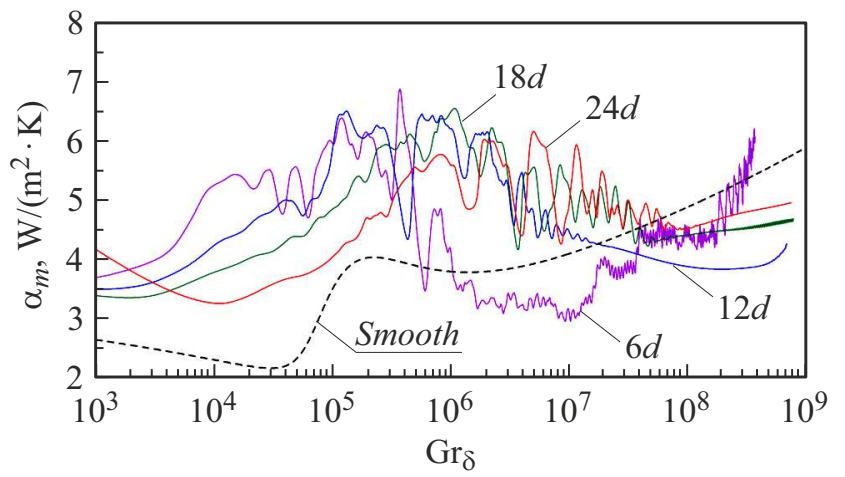

Рис. 3. Влияние продольного шага в расположении выступов на зависимость интегрального коэффициента теплоотдачи от характерного числа Грасгофа (сплошные линии) в сопоставлении с данными для гладкой пластины (штриховая линия).

турбулентного перехода. Полученные в настоящих расчетах для пластины с выступами на стадии достаточно развитой турбулентности $\left(\mathrm{Gr}_{\delta}>10^{6}\right)$ зависимости $\alpha_{m}\left(\mathrm{Gr}_{\delta}\right)$ существенно немонотонны, что отражает факт наличия низкочастотных колебаний. Анализ временнб́х изменений в рассчитанных картинах течения показал, что эти колебания обусловлены внутренней неустойчивостью крупномасштабных вихревых структур, формирующихся в следе за каждым препятствием. Интересно, что в случае примененной модели рейнольдсовых напряжений гидродинамическая неустойчивость этих крупных структур предсказывается даже при наложении условий симметрии, существенно ослабляющих свободу поперечных (трансверсальных) перемещений частиц жидкости. Проведенное осреднение теплового потока 
по ограниченному, выделенному для расчетов участку нагреваемой пластины лишь частично сглаживает влияние предсказываемых неустойчивостей на временну́ю эволюцию интегральной теплоотдачи. В целом же по приведенным на рис. 3 данным можно заключить, что в интервале чисел Грасгофа от $10^{6}$ до $2 \cdot 10^{7}$ турбулентная теплоотдача от пластины с выступами, расположенными с большим шагом по вертикали, существенно выше, чем в случае гладкой пластины. Максимальный выигрыш в теплоотдаче от использования массива выступов достигается в начале фазы развитой турбулентности при $s_{2} \approx 20 d$ и составляет около $80 \%$.

\section{Конфликт интересов}

Авторы заявляют, что у них нет конфликта интересов.

\section{Список литературы}

[1] В.И. Терехов, Т.В. Богатко, А.Ю. Дьяченко, Я.И. Смульский, Н.И. Ярыгина, Теплообмен в дозвуковых отрывных потоках (Изд-во НГТУ, Новосибирск, 2016).

[2] С.В. Гувернюк, А.Ю. Чулюнин, Письма в ЖТФ, 45 (17), 43 (2019). DOI: 10.21883/PJTF.2019.17.48224.17887 [S.V. Guvernyuk, A.Yu. Chulyunin, Tech. Phys. Lett., 45 (9), 894 (2019). DOI: 10.1134/S1063785019090062].

[3] С.А. Исаев, А.Б. Мазо, Д.В. Никущенко, И.А. Попов, А.Г. Судаков, Письма в ЖТФ, 46 (21), 18 (2020). DOI: 10.21883/PJTF.2020.21.50190.18454 [S.A. Isaev, A.B. Mazo, D.V. Nikushchenko, I.A. Popov, A.G. Sudakov, Tech. Phys. Lett., 46 (11), 1064 (2020). DOI: $10.1134 / \mathrm{S} 1063785020110073$ ].

[4] И.А. Попов, Интенсификация теплообмена. Гидродинамика и теплообмен внешних и внутренних вертикальных течений с интенсибикацией (Центр инновационных технологий, Казань, 2007).

[5] T. Tsuji, Y. Nagano, Int. J. Heat Mass Transfer, 31 (8), 1723 (1988). DOI: 10.1016/0017-9310(88)90284-0

[6] T. Tsuji, T. Kajitani, T. Nishino, Int. J. Heat Fluid Flow, 28 (6), 1472 (2007). DOI: 10.1016/j.ijheatfluidflow.2007.04.021

[7] E.M. Smirnov, A.M. Levchenya, V.D. Zhukovskaya, Int. J. Heat Mass Transfer, 144 (12), 118573 (2019). DOI: $10.1016 /$ j.ijheatmasstransfer.2019.118573

[8] M.R. Kotikova, A.M. Levchenya, M.A. Zasimova, E.M. Smirnov, J. Phys.: Conf. Ser., 1565, 012082 (2020). DOI: 10.1088/1742-6596/1565/1/012082

[9] M. Fujii, Heat Transfer - Asian Res., 36 (6), 334 (2007). DOI: $10.1002 / \mathrm{htj} .20168$

[10] M. Imbriale, M. Panelli, G. Cardone, Quant. InfraRed Thermogr. J., 9 (1), 55 (2012).

DOI: $10.1080 / 17686733.2012 .681881$

[11] M.M. Naserian, M. Fahiminia, H.R. Goshayeshi, J. Mech. Sci. Technol., 27 (7), 2191 (2013). DOI: $10.1007 / \mathrm{s} 12206-013-0535-7$

[12] M.Z. Abedin, T. Tsuji, Y. Hattori, Int. J. Heat Mass Transfer, 52 (19-20), 4525 (2009). DOI: 10.1016/j.ijheatmasstransfer.2009.03.061

[13] A.G. Abramov, E.M. Smirnov, V.D. Goryachev, Fluid Dyn. Res., 46 (4), 041408 (2014).

DOI: $10.1088 / 0169-5983 / 46 / 4 / 041408$
[14] А.М. Левченя, С.Н. Трунова, Е.В. Колесник, Науч.-техн. ведомости СПбГПУ. Физ.-мат. науки, 13 (2), 27 (2020). DOI: $10.18721 /$ JPM.13203

[15] D.C. Wilcox, Turbulence modeling for CFD, 2nd ed. (DCW Industries, La Canada, California, 1998). 